\title{
THE BACTERIAL OXIDATION OF AMMONIA IN THE SEA
}

\author{
By C. P. Spencer \\ Marine Biology Station, University College of North Wales, \\ and The Plymouth Laboratory
}

(Text-figs. I-3)

The final regeneration stages of the nitrogen cycle in the sea are believed to consist of the bacterial oxidation of ammonium-nitrogen to nitrite and finally nitrate-nitrogen. There have been many attempts to demonstrate the presence in the sea of marine counterparts to the terrestrial Nitrosomonas and Nitrobacter. (See Zobell, 1946, for bibliography of the earlier work.) Bacteria which can oxidize either ammonia or nitrite are easily demonstrated in samples of bottom material or water contaminated by land drainage or bottom deposits. On the other hand, there has been general failure to demonstrate the presence of such species in the upper layers of the open ocean.

Nevertheless, oceanographical evidence suggests that considerable nitrification occurs in these upper layers. There is no evidence that, under natural conditions, purely chemical catalysis occurs to any appreciable extent. The photo-oxidation of ammonia in sea water by ultra-violet light has been demonstrated (Zobell, I933), but it is unlikely that this can be important except to a depth of about $\mathrm{I} \mathrm{m}$. The failure to demonstrate the presence of ammonia-oxidizing bacteria in the middle layers of open ocean water therefore leaves a serious gap in our knowledge of the nitrogen cycle in the sea. Zobell (I946) has suggested that some marine nitrifiers may exist which are of a very different physiological character from the strictly autotrophic terrestrial species, and that failure to demonstrate their presence might perhaps therefore be due to the use of unsuitable culture methods.

The present paper reports the results obtained during an investigation of the nitrifying behaviour of the surface water from the International Hydrographical Station E I. These studies were commenced whilst working at the Plymouth Laboratory and were continued and extended at the Marine Biology Station of the University College of North Wales, Menai Bridge.

\section{Methods}

All cultures were grown in $250 \mathrm{ml}$. Pyrex conical flasks capped with small beakers. The medium used was essentially that employed by Carey (I938). For initial enrichment cultures Io $\mathrm{g}$ of acid-washed fine sand and I $\mathrm{g}$ calcium 
carbonate were dispensed in each flask and sterilized by autoclaving at Io $\mathrm{lb}$. for $20 \mathrm{~min} ; 40 \mathrm{ml}$. samples of sea water were added followed by aseptic additions of phosphate $\left(\mathrm{O} \cdot \mathrm{I} \times \mathrm{IO}^{-3} \mathrm{M}\right)$ and ammonium chloride $\left(2.0 \times \mathrm{IO}^{-3} \mathrm{M}\right)$ from sterile stock solutions of the required strength. For making sterile media for subsequent cultures, $40 \mathrm{ml}$. of aged sea water were added to each flask, together with the sand and calcium carbonate, and the whole sterilized by autoclaving at $5 \mathrm{lb}$. for $30 \mathrm{~min}$. All cultures were incubated at $22^{\circ} \mathrm{C}$ in the dark.

Experience showed that little nitrification occurred without a solid phase in the medium. Further tests demonstrated that sand alone was not very effective as a solid phase. Carbonate was much more effective, and in the presence of calcium carbonate an addition of sand had little further effect. In the later experiments the sand was therefore omitted.

The progress of ammonia oxidation was followed by withdrawing samples at intervals and estimating for nitrite using the method of Rider \& Mellon (1946). The cultures were agitated before samples were withdrawn to suspend the carbonate evenly. After development of the colour, the intensity was read by means of a Harvey absorptiometer using Ilford Filters no. 604. Nitrite was determined by means of a calibration curve prepared by assaying solutions of sodium nitrite of known strengths containing suspended calcium carbonate in the same proportions as in the cultures. The carbonate dissolves in the acid medium used for the diazotization, but this and the final coupling reaction are $\mathrm{pH}$ sensitive, and the calibration curve was therefore prepared using samples analogous to those obtained from cultures.

\section{EXPERIMENTAL}

In the preliminary experiments the nitrifying behaviour of surface water from the International Hydrographical Station E I was investigated by setting up enrichment cultures. Surface water was collected after the development of the thermocline and dispensed in $40 \mathrm{ml}$. amounts in sterile flasks containing sand and calcium carbonate with additions of phosphate and ammonium chloride. Periodic sampling showed that no formation of nitrite could be detected in this series of cultures in up to 80 days' incubation, and if any oxidation of ammonia was occurring it must have been extremely slow. No tests were made for the formation of nitrate since most previous investigators agreed that nitrite formation became maximal before appreciable oxidation to nitrate occurred (von Brant, Rakestraw \& Renn, 1937).

These preliminary results largely confirmed the much earlier findings of Harvey (1926) with surface-water samples from the same station. They further suggested that it was not solely the lack of a solid phase in the culture medium that was inhibiting ammonia oxidation as suggested by Cooper (1948). 
In a further attempt to set up enrichment cultures a series of water samples were incubated as before except that an additional addendum of $\mathrm{I} \mathrm{ml}$. of a uni-algal (but not bacteria free) stock culture of a diatom was made to each flask. After an initial induction period of 35 days nitrite formation commenced in all cultures of this series and continued vigorously until a maximum value was reached after some 60 days. The observation that the presence of plankton material allowed nitrification to proceed was reminiscent of the results of Carey (1938).

Another series of enrichment cultures was set up to identify the origin of the ammonia-oxidizing bacteria. These cultures showed that no nitrite formation occurred if the sea-water samples were sterilized by autoclaving regardless of whether the diatom addendum was sterile or not. Further, with freshly collected unsterilized water samples, the progress of nitrite formation showed little difference whether the diatom suspension together with associated bacteria was added in a fully viable condition or after having been sterilized by autoclaving. It therefore seemed certain that ammonia-oxidizing bacteria were present in the water samples and not in the crude diatom culture. Further confirmation of this was obtained by the use of a bacteria-free culture of a marine diatom. The addition of this also allowed nitrite formation to occur in non-sterile samples of the sea water, whereas nitrite formation could not be detected after 80 days' incubation in any control culture set up with sterilized sea water or without additions of the diatom suspension.

Attempts were next made to subculture the nitrifying organism. I ml. samples of the initial enrichment cultures described above were transferred to sterile medium without the addition of the diatom suspension. The formation of nitrite proceeded in all the subcultures without the marked induction period observed in the original enrichment cultures but at a much reduced rate. In subsequent subcultures the rate of nitrite formation was further successively reduced. Progress curves for the production of nitrite in initial enrichment cultures and in a subsequent subculture are shown in Fig. I. The concentration of nitrite in the enrichment cultures varied exponentially with time. This is typical of an autocatalytic reaction and is the effect that might be expected with a proliferating catalytic organism. On the other hand, the concentration of nitrite in the subsequent subcultures was directly proportional to the time of incubation.

An investigation was made of the effect of variations in the size of inocula on the kinetics of nitrite production in subcultures without the addition of diatoms. Within limits, the rate of nitrite production varied directly with the size of inocula and remained linear. With the largest inocula, however, the production of nitrite, though initially linear, tended to give way to an exponential rate. The kinetics of nitrite formation in subcultures without added diatoms and the results of variations in the inocula size in such subcultures 
suggested the presence of a non-proliferating but enzymically active bacterial population.

The addition of a sterilized diatom suspension to a subculture inhibited the production of nitrite for periods of up to 30 days. Thereafter rapid exponential formation of nitrite occurred. A hot-water extract of diatoms from which the insoluble debris had been removed by centrifuging was considerably less inhibitory, the induction period in this case lasting only 2 or 3 days. Such extracts seemed equally effective as whole cells in ultimately promoting exponential nitrite formation in subcultures. In control experiments only

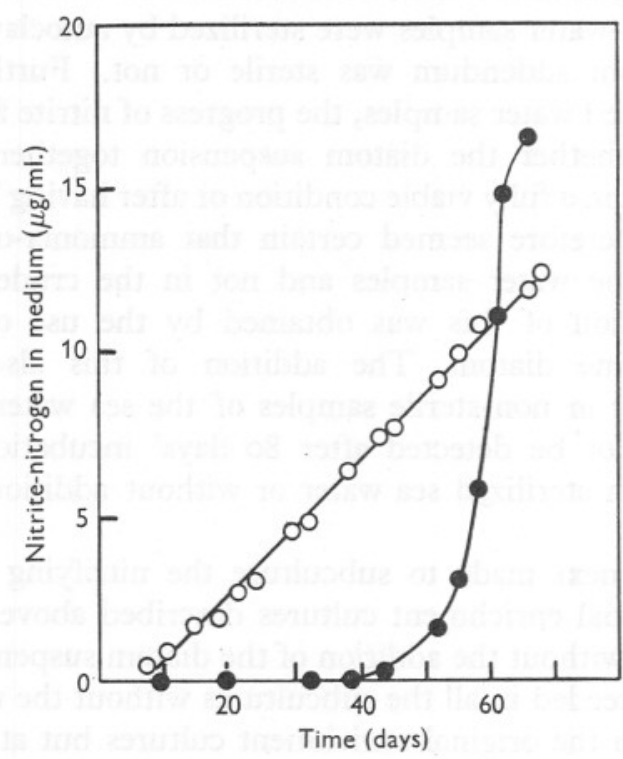

Fig. I. Progress curve for nitrite production in medium made up with E I water. Initial enrichment culture with addition of diatoms; - $-\mathrm{O}-$, subculture without addition of diatoms.

negligible production of nitrite occurred in cultures containing sterile diatom suspensions or hot-water diatom extracts without the addition of ammoniumnitrogen. It therefore seemed probable that the ammonia-oxidizing bacteria in the water samples needed for growth some factor or factors supplied by the diatoms.

An examination was made of the effects of the initial concentration of ammonia on the rate of nitrite production in non-proliferating cultures. Variable results were obtained, and it proved impossible to obtain repeatable data for a concentration-rate curve. However, the results indicated that initial concentrations of over $0.01 \mathrm{M}$ ammonium-nitrogen were increasingly inhibitory to the rate of nitrite formation. 
Quastel, Scholefield \& Stevenson (I952) have reported the direct production of nitrite from organic nitrogen without the intermediate formation of ammonia. The results of control cultures and the recoveries of nitrogen as nitrite, which agreed well with the original addition of ammonia, made it unlikely that the nitrite could in any case be formed entirely from the organic nitrogen of the diatoms. It remained possible that appreciable nitrite might be formed directly from organic nitrogen synthesized by the heterotrophic bacterial flora present. All attempts to demonstrate the presence of any organism capable of producing nitrite from pyruvic acid oxime gave negative results.

The experiments were continued at the Marine Biology Station, Menai Bridge. An investigation was first made of the nitrifying behaviour of freshly collected water from the Menai Straits. These water samples showed vigorous formation of nitrite when incubated with calcium carbonate and suitably enriched with phosphate and ammonium-nitrogen. Furthermore, when subcultures were made from these enrichment cultures to sterile media made up from Menai Straits water without the addition of diatoms, the exponential production of nitrite persisted without diminishing throughout numerous successive subcultures. This behaviour was in marked contrast to that shown by cultures set up in E I water. The sea water used in these and all subsequent subcultures was 'aged' water which had been filtered through Whatman no. 2 filter-paper immediately after collection and then allowed to stand for at least I month in the dark.

These results agreed with those reported by many previous workers, for there seems to be general agreement that nitrifying bacteria occur in coastal waters contaminated by land drainage and bottom deposits. In conjunction with the results reported above, which demonstrated the presence of ammoniaoxidizing bacteria in E I water which apparently required for growth a factor or factors in diatoms, there were two possible interpretations of the observed experimental observations. Either the ammonia-oxidizing organisms which were present in the two waters were of two types with differing nutritional requirements or, alternatively, the Menai Straits water contained the factors supplied to EI water by a diatom suspension. Experiments were therefore made to investigate these possibilities.

Cultures were set up in sterile media of identical composition made up with either aged Menai Straits water or a sample of aged E I water (membranefiltered immediately after collection). These cultures, together with the relevant controls, were inoculated from an enrichment culture set up with Menai Straits water. The results of such an experiment are shown in Fig. 2. It will be seen that the cultures in medium made up with Menai Straits water exhibited an exponential production of nitrite and therefore presumably grew well. In contrast, the cultures in medium made up with E I water exhibited a slow linear production of nitrite. This behaviour was similar to the subcultures from the original EI enrichment cultures. This similarity was 
emphasized by the subsequent observation that the addition of a sterile diatom suspension allowed an exponential production of nitrite in cultures made up with EI water and inoculated from Menai Straits enrichment cultures. It therefore seemed likely that there was a difference between the two water masses with respect to their ability to support the growth of the ammonia-oxidizing bacteria, there being no evidence to suggest that the ammonia-oxidizing bacteria from the two localities had different nutritional requirements.

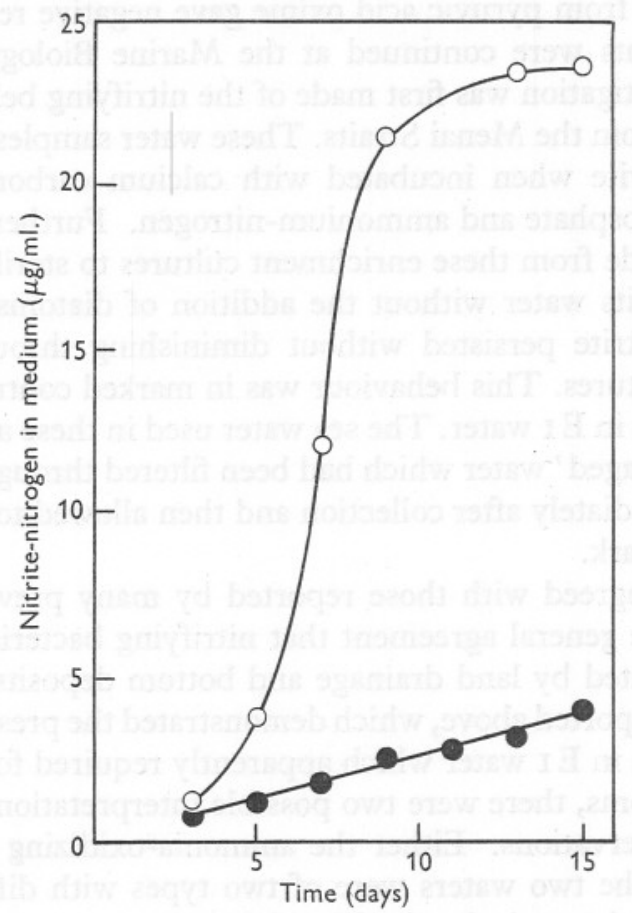

Fig. 2. Progress curve for nitrite production after subcultures from Menai Straits enrichment culture. - $-\mathrm{O}-$, Medium made up with Menai Straits water; - $-0-$, medium made up with E I water.

An examination of the two waters showed markedly different contents of iron (estimated by the method of Cooper, 1935). The sample of E I water used contained a barely detectable amount of iron, whereas water from the Menai Straits had an iron content of the order of $20-30 \mu \mathrm{g} / 1$. Meiklejohn has shown that the terrestrial species of Nitrosomonas and Nitrobacter require considerable amounts of iron for growth. In addition, it seemed certain that some iron was being added with the diatoms since these had been grown in iron-rich medium and are known to accumulate considerable quantities of iron. It therefore appeared possible that variations in the iron content might account 
for the variations in the ability of the two waters to support the growth of the marine nitrifiers. Experiments showed (Fig. 3) that the addition of iron to subcultures in medium made up with E I water allowed an exponential production of nitrite. The concentrations of nitrite in Fig. 3 are plotted on a logarithmic scale and the slope of the graphs is presumably a measure of the growth rate of the nitrifying bacteria. It will be seen that the additions of iron to E I water allowed growth at a rate comparable to that in Menai Straits water.

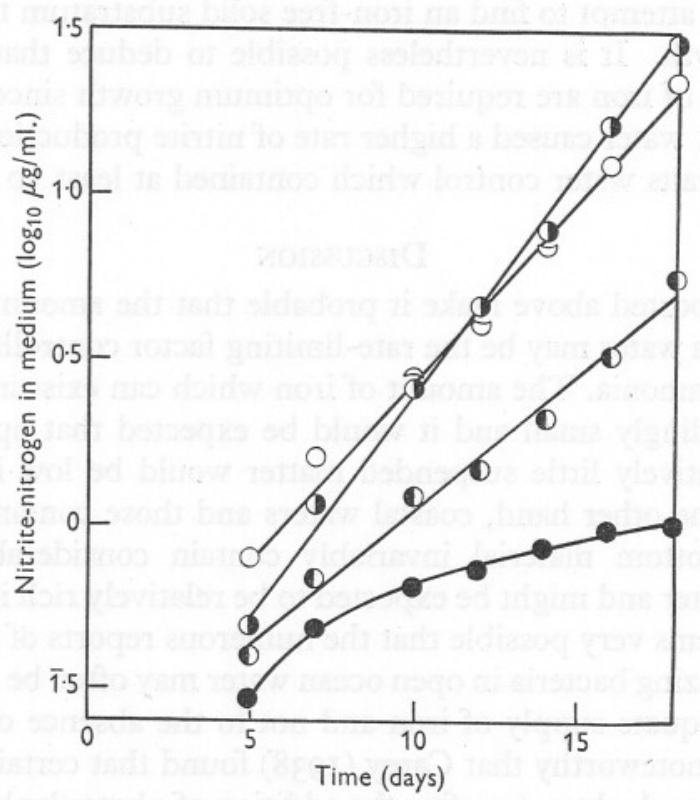

Fig. 3. Progress curves for nitrite production after subcultures from a Menai Straits enrichment culture. $\longrightarrow-$, E I water medium, no additions; - $-\mathrm{O}$, Menai Straits water medium, no additions; - D-D-, E I water medium with addition of diatoms; $-\infty-$ - E I water medium with addition of $100 \mu \mathrm{g}$./1. iron.

Medium made up with EI water with the addition of iron supported growth of the ammonia-oxidizing bacteria in numerous successive subcultures and after ten subcultures there was no indication of any diminished growth rate. Subsequent successive subcultures without the addition of iron showed decreasing rates of growth and after three or four subcultures of this type the production of nitrite again became proportional to time and characteristic of a non-proliferating bacterial population. It is noticeable that several subcultures are necessary for the effect to become apparent in this case, in contrast to the linear production of nitrite in the first subcultures from Menai Straits water to E I water without the addition of a diatom suspension. This is probably due to the much lower content of iron in the latter parent cultures. 
The iron content of Menai Straits water can be greatly reduced by filtration through a membrane filter. The growth of the ammonia-oxidizing bacteria in medium made up with this filtered water was similar to that observed in medium made up with EI water.

It has not been found possible to determine the optimum concentration of iron for the growth of the marine nitrifiers since analysis showed relatively considerable quantities of iron in the calcium carbonate, it being impossible to assess how much of this would be available to the bacteria. No success was obtained in an attempt to find an iron-free solid substratum that would allow optimum growth. It is nevertheless possible to deduce that relatively high concentrations of iron are required for optimum growth since the addition of Io० $\mu \mathrm{g} / 1$. to E I water caused a higher rate of nitrite production than occurred in a Menai Straits water control which contained at least $30 \mu \mathrm{g} / 1$. (Fig. 3).

\section{Discussion}

The results reported above make it probable that the amount of iron present in a body of sea water may be the rate-limiting factor controlling the bacterial oxidation of ammonia. The amount of iron which can exist in solution in sea water is exceedingly small and it would be expected that open ocean water containing relatively little suspended matter would be low in available iron content. On the other hand, coastal waters and those contaminated by land drainage or bottom material invariably contain considerable amounts of suspended matter and might be expected to be relatively rich in available iron. It therefore seems very possible that the numerous reports of failure to detect ammonia-oxidizing bacteria in open ocean water may often be due to failure to provide an adequate supply of iron and not to the absence of the catalysing bacteria. It is noteworthy that Carey (1938) found that certain waters naturally rich in phytoplankton (or after the addition of phytoplankton townetting) allowed the vigorous oxidation of ammonia, whereas in similar samples containing a preponderance of zooplankton little nitrification occurred. Judging by the result obtained by Cooper (I935) on the iron content of phytoplankton and zooplankton, Carey's observation could be due to the relatively higher iron content of the phytoplankton.

If this interpretation is correct, considerable bacterial oxidation of ammonia may occur in the upper layers of the open ocean as suggested by the oceanographical evidence. The actual rate of oxidation in nature would be expected to be much lower than that observed in culture, due not only to relatively low levels of iron but also to low substrate concentrations. It is obvious, however, that such waters might fail to show appreciable nitrifying activity if cultured without the addition of iron. A wide survey of the nitrifying activity of freshly collected open ocean waters using the culture techniques described in this paper is required to test this possibility.

Details of the identity and nature of the catalysing organism must await its 
isolation. Several previous workers have reported the failure of attempted isolation studies. Waksman, Hotchkiss \& Carey (1933) noted the formation of nitrite on silica gel plates inoculated from their enrichment cultures, but apparently no observable growth occurred. This is reminiscent of the production of nitrite without proliferation of the catalysing organism noted during the present studies. It seems probable therefore that any silica gel medium used in isolation studies with the marine nitrifiers will have to be well enriched with iron. There is, however, no evidence to suggest that the marine nitrifiers are of different physiological type to their terrestrial counterparts. The results obtained by von Brandt et al. (I937), by Carey (I938), and in the present studies show that the onset of the oxidation of ammonia is delayed in cultures containing organic matter. Nitrosomonas is a strict autotroph and its growth is inhibited by organic matter. During the induction period presumably the heterotrophic flora present is removing the inhibitory organic matter. Furthermore, the observation of von Brant et al. that oxidation of nitrite does not commence until all the ammonia has been oxidized is typical of the behaviour of Nitrobacter, the growth of which is inhibited by quite low concentrations of ammonia. The inhibition of ammonia oxidation, with increasing ammonia concentrations of the order noted in the present studies, is typical of Nitrosomonas.

It is interesting that the optimum iron concentration in culture lies well above the solubility of the ferric ion. Similar results were obtained by Meiklejohn (I953). Working with the terrestrial Nitrosomonas she reported optimum iron concentrations of the order of $6 \mathrm{mg} / 1$. At the high $\mathrm{pH}$ values employed for culture these iron concentrations are certainly in excess of the solubility of iron. These observations and the present studies suggest, therefore, that these organisms are capable of, and may be dependent on, utilizing particulate iron. The nitrifying bacteria show a very marked tendency to attach themselves to particles of the solid phase in the culture medium. Lees \& Quastel (I946) have shown that ammonia is not oxidized in soil cultures unless it is absorbed on to the surface of the soil colloids. The adherence tendencies of these bacteria may thus be necessitated by their method of mobilization of ammonia. If, however, considerable quantities of iron are required, the bulk of which is in particulate form, it is difficult to see how this could be mobilized unless physical contact between the cells and the particles was achieved. The attachment propensities of these organisms may thus be due in part to the necessity of their mobilizing iron in particulate form.

Part of the work reported above was performed at the Plymouth Laboratory. It is a great pleasure to thank the Director and Staff for making freely available the facilities of the laboratory, especially to Dr H. W. Harvey, F.R.S., for his continual interest and encouragement, and $\mathrm{Mr} \mathrm{F}$. A. J. Armstrong for collecting water samples. 


\section{SUMMARY}

The failure of a sample of E I water to oxidize added ammonium-nitrogen to nitrite-nitrogen has been shown to be due, not to the absence of catalytic bacteria in the water, but rather to the lack of suitable nutrient conditions for proliferation of the nitrifying bacteria.

The addition of sterile diatoms or sterile hot water extracts of diatom cells to E I water allowed the proliferation of the nitrifying organisms with concurrent oxidation of ammonium-nitrogen to nitrite-nitrogen.

Water from the Menai Straits allows the proliferation of its naturally occurring nitrifying flora without additions of diatom material. These organisms, however, will not grow in E I water unless diatom material is added.

Additions of iron allow the nitrifying bacteria from Menai Straits water to grow in EI water. These additions of iron have a quantitative effect comparable to additions of diatom material.

It is suggested that the many previous failures to demonstrate the presence of nitrifying bacteria in the middle layers of open ocean waters may be duenot to their absence but to failure to supply sufficient iron for appreciable growth to occur in culture. This may bring the bacteriological evidence more in line with the oceanographical evidence which suggests that considerable formation of nitrite occurs in these layers.

\section{REFERENCES}

BRANT, T. von, RAKestraw, N. W. \& RenN, C. E., I937. The experimental decomposition and regeneration of nitrogenous organic matter in sea water. Biol. Bull., Woods Hole, Vol. 72, pp. 165-75.

CAREY, C. L., I938. The occurrence and distribution of nitrifying bacteria in the sea. F. mar. Res., Vol. I, pp. 29I-304.

Cooper, L. H. N., 1935. Iron in the sea and in marine plankton. Proc. Roy. Soc. B, Vol. II8, pp. 419-38.

1948. Particulate ammonia in sea water. F. mar. biol. Ass. U.K., Vol. 27, pp. $322-5$.

HARVEY, H. W., I926. Nitrate in the sea. F. mar. biol. Ass. U.K., Vol. I4, pp. 7 I-88.

LeES, H. \& QUASTEL, J. H., I946. Biochemistry of nitrification in soil. The site of soil nitrification. Biochem. F., Vol. 40, pp. 815-23.

Meiklejohn, J., I953. Iron and the nitrifying bacteria. F. gen. Microbiol., Vol. 8, pp. $58-65$.

Quastel, J. H., Scholefield, P. G. \& Stevenson, J. W., I952. Oxidation of pyruvic acid oxime by soil organisms. Biochem. F., Vol. 5I, pp. 278-84.

Rider, B. F. \& Mellon, M. G., 1946. Colorimetric determination of nitrites. Industr. Engng Chem., Vol. I8, pp. 96-9.

WAKSMAN, S. A., HotchKISS, M. \& CAREY, C. L., I933. Marine bacteria and their role in the cycle of life in the sea. II. Bacteria concerned in the cycle of nitrogen in the sea. Biol. Bull., Woods Hole, Vol. 65, pp. 137-65.

Zobell, C. E., 1933. Photochemical nitrification in sea water. Science, Vol. 77, pp. 27-8.

— I946. Marine microbiology. 240 pp. Waltham, Mass.: Chron. bot. Co. 